\title{
Facial nerve palsy in the course of herpes zoster infection - challenges in the treatment and prognosis
}

\author{
Maja Pietrzak', Maria Śpiewak-Pokorska1,2, Magdalena Pluta ${ }^{1,2}$, Magdalena Marczyńska ${ }^{1,2}$ \\ ${ }^{1}$ Pediatric Infectious Diseases Unit, Regional Hospital of Infectious Diseases in Warsaw, Warsaw, Poland \\ 2Department of Children's Infectious Diseases, Medical University of Warsaw, Warsaw, Poland
}

\section{ABSTRACT}

Facial nerve palsy occurs in paediatric population with the incidence of 4.2-15.3/100 000. In most cases etiology remains unknown (Bell's palsy - idiopathic facial nerve palsy). Infectious agents account for around $25 \%$ of cases. This paper describes 2 cases of facial nerve palsy due to herpes zoster infection: a 9-year-old girl with vesicular lesions on the right side of the neck and face with concomitant right sided facial nerve palsy, and a 16-year-old boy ${ }^{*}$ with lesions located in the left external auditory meatus who developed Ramsay-Hunt syndrome. The course of the disease and response to treatment in both patients was significantly different.

KEY WORDS:

Ramsay-Hunt syndrome, facial palsy, Herpes zoster.

* Both patients and their legal guardians provided oral consent for this publication.

\section{INTRODUCTION}

Herpes zoster is a rare and usually mild disease in the paediatric population. Incidence in children $<10$ years old is around 0.74 cases/1000 persons per year [1]. Rarely it is complicated by facial nerve paralysis, particularly in the form of Ramsay-Hunt syndrome (RHS). Ramsay-Hunt syndrome is the manifestation of Herpes zoster oticus which is defined by the triad of symptoms including ipsilateral ear pain, vesicles in the external auditory canal, and facial nerve palsy [2]. It has a reported incidence of 2.7/100 000 in children and is associated with acute facial nerve paralysis [3]. It is the second most common cause of atraumatic facial palsy. Typically facial palsy appears between the second and fifteenth day after first vesicular lesions, and only in $7.4 \%$ of patients nerve VII palsy was the first symptom to be observed [4].

\section{CASE 1}

On the $16^{\text {th }}$ of January, 2021, a 15-year-old boy presented to the Emergency Room (ER) of Children's Hospital in Dziekanów Leśny with pain in the left ear and ptosis of the left eyelid. He was diagnosed with facial nerve palsy, but his parents refused diagnostic hospitalisation in the paediatric neurological ward. Upon discharge he was ordered methylprednisolone $0.3 \mathrm{mg} / \mathrm{kg}$ body weight (morning dose) $+0,15 \mathrm{mg} / \mathrm{kg}$ body weight (afternoon dose), acyclovir $10 \mathrm{mg} / \mathrm{kg}$ body weight $5 \times$ /day for 5 days (Herpes simplex virus dosing), artificial tears and physical therapy. In the outpatient setting he was tested for Lyme Disease, which was ruled out (antibodies class M and G negative). On the $20^{\text {th }}$ of January he presented to the ER of the Bogdanowicz Memorial Hospital in Warsaw. He was examined by consulting neurologist who diagnosed him with facial nerve palsy, House-Brackmann grade 4 . Head CT (computed tomography) showed no abnormalities. Consulting ENT (ear nose and throat) specialist

\section{ADDRESS FOR CORRESPONDENCE:}

Maja Pietrzak, Pediatric Infectious Diseases Unit, Regional Hospital of Infectious Diseases in Warsaw, Warsaw, Poland, e-mail: maja.pietrzak@gmail.com. 
reported no further findings. He was prescribed prednisone $1 \mathrm{mg} / \mathrm{kg}$ body weight, artificial tears, topical dexapanthenolum, multivitamin supplements and rehabilitation. On the $26^{\text {th }}$ of January he presented to the ER of the latter hospital because of mixed purulent-hemorrhagic effusion from the left ear. He was consulted by dermatologist and ENT specialist who reported bloody crusts in the left ear lobe and suggested repeating acyclovir therapy due to the suspicion of Herpes simplex virus (HSV) or Varicella-zoster virus (VZV) aetiology. The patient was diagnosed with Ramsay-Hunt Syndrome. Additionally to the latter treatment he was prescribed oral Acyclovir $8 \mathrm{mg} / \mathrm{kg}$ body weight $3 \mathrm{x} /$ day for 7 days. On $2^{\text {nd }}$ of February (18 $8^{\text {th }}$ day since the onset of symptoms) due to no improvement in the facial nerve function, he was referred to our Paediatric Department. He had no history of varicella or varicella immunisation, no history of previous hospitalisations or infections in the past few weeks and did not suffer from any chronic conditions. Parents reported allergy to amoxicillin.

On admission he was in good general condition. $\mathrm{He}$ had no nausea, vertigo, balance disorders, nor hearing loss. Physical examination revealed acne, follicular keratosis and melanocytic naevus on the left lower limb, in otoscopy left tympanic membrane was reddish and bloody crusts were to be find on the border of ear canal and auricle, behind the right tympanic membrane there was small amount of transparent fluid. In neurological examination, facial nerve palsy grade IV/V House-Brackmann scale was found. In laboratory testing leukocytosis $(24.9 \times 1000 / \mathrm{mm} 3)$ with majority of neutrophils $(21.92 \times$ $1000 / \mathrm{mm} 3$ ) was found. Serological testing revealed positive antibodies class G for VZV.

Intravenous acyclovir in the dose of $10 \mathrm{mg} / \mathrm{kg}$ body weight $3 \times$ /day for 5 days was administered. Additionally, physical rehabilitation, massages, kinesiotaping and bioptron lamp irradiation were implemented.

During hospitalisation, transient asymmetry in the vesicular breath sounds was found. Chest X-ray revealed no abnormalities. Small progress in the recovery of facial nerve function was noted, including improvement upon eye closing, House-Brackmann grade III. Repeated complete blood count was in normal range.

After 5 days of hospitalisation he was discharged home to continue treatment with vitamin supplements and rehabilitation scheduled in the outpatient setting.

At 3 month telephone call follow-up patient reported return of full eye closure but minimal movement ability in the area of the mouth and forehead. He remains in rehabilitation in the outpatient setting.

\section{CASE 2}

On the $6^{\text {th }}$ of February 2021, a 9-year-old girl presented to the hospital with right-sided facial nerve palsy. On the $31^{\text {st }}$ of January she noticed vesicular rash on the right side of her face and neck with accompanying ear pain. She also complained of sporadic cough and loss of appetite. On the $3^{\text {rd }}$ of February general practitioner prescribed her amoxicillin due to suspected bacterial infection. Around 24 hours before admission the patient noticed change of taste and numbness of the face followed by palsy. She had a history of varicella at the age of four years. In the early childhood she was being diagnosed for urolithiasis and remained under the supervision of the nephrologist. She had a history of allergy to axetil cefuroxime and recurrent ear infections - with the last episode about one year ago.

On admission she was in good general condition, without nausea and vertigo. Physical examination revealed peripheral facial nerve palsy House-Brackmann grade III/IV and Herpes zoster lesions on the right side of the neck and under the right eye.

Laboratory tests results were within normal reference range. Due to the epidemiological situation she was tested for SARS-CoV-2: antigen test result was negative, antibodies class IgG were positive. Serological testing for Lyme Disease was performed and the diagnosis was ruled out.

Treatment with amoxicillin was continued, additionally she was administered acyclovir $500 \mathrm{mg} / \mathrm{m}^{2}$ intravenously (i.v.) 3 times a day for 7 days, dexamethasone $0,5 \mathrm{mg} / \mathrm{kg}$ body weight/day i.v., B1 and B6 vitamin supplements and intravenous fluids.

Due to reported abdominal pain, proton-pump inhibitors (PPIs) were introduced. Physical rehabilitation and phototherapy were started.

During hospitalisation patient experienced one episode of anxiety attack with heart palpitations, accelerated heart rate and elevated blood pressure which resolved without additional medication. Echocardiogram showed no abnormalities. The dose of steroids was gradually tapered down.

Improvement in the motor function of the face was observed - upon discharge facial palsy was assessed as House-Brackmann grade II. After 7 days of hospitalisation she was discharged home with gradual tapering down the steroid dose, vitamin supplements and rehabilitation in outpatient setting.

At 3 month telephone call follow up patient reported full return of facial nerve function. Due to persisting ear pain, she remains under ENT's care.

\section{DISCUSSION}

Facial nerve palsy is defined as a rapid loss of movement ability affecting usually one side of the face. It is a relatively rare symptom in children. The vast majority of the cases is of unknown aetiology (idiopathic facial nerve palsy - Bell's palsy). Herpes simplex virus is implicated as the most frequent infectious causative agent of this symptom, others are for example Epstein-Barr virus, Borrelia burgdorferi and Varicella-Zoster virus.[1] 
TABLE 1. House Brackmann scale (personal materials)

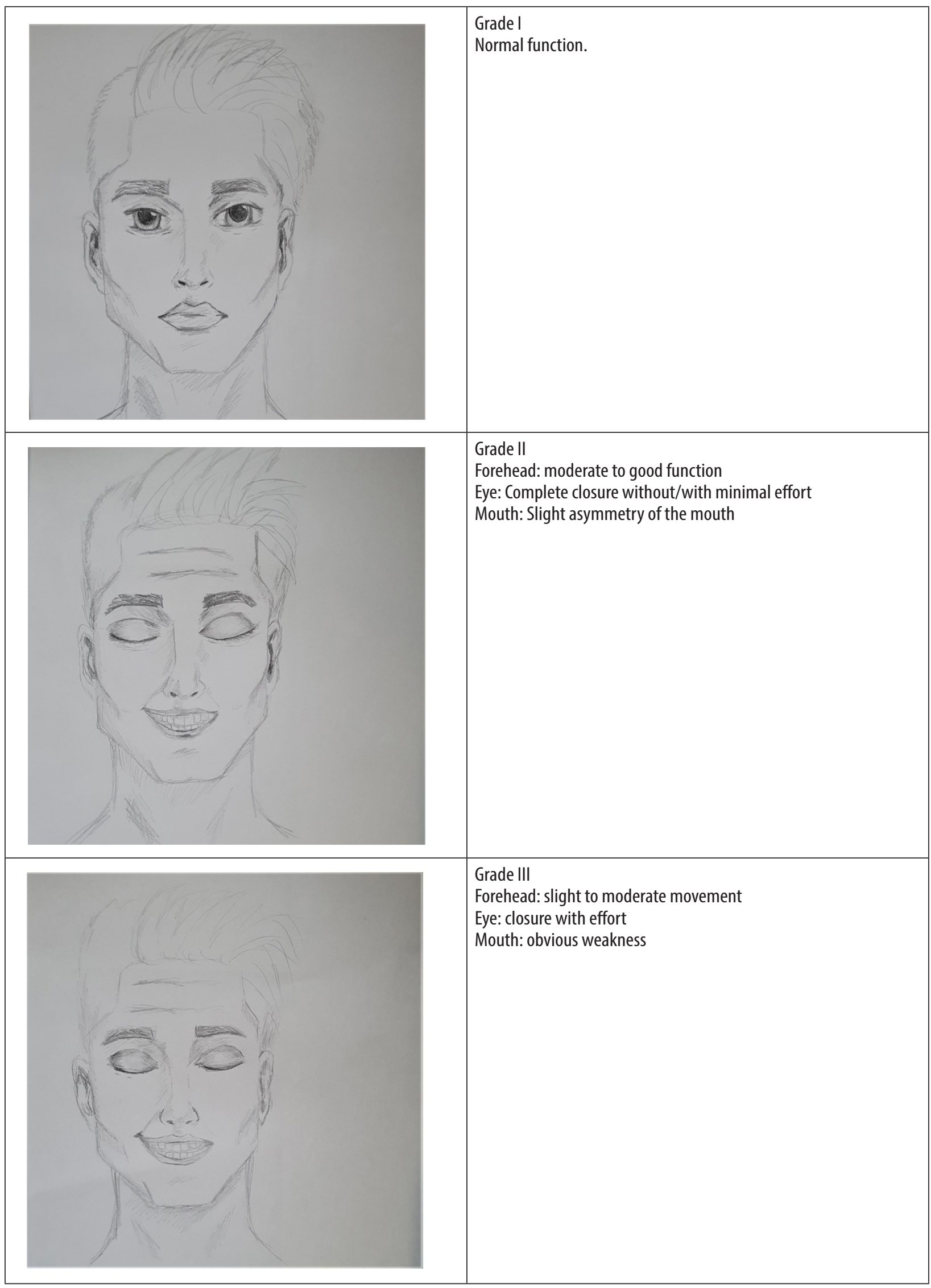


TABLE 1. Cont.

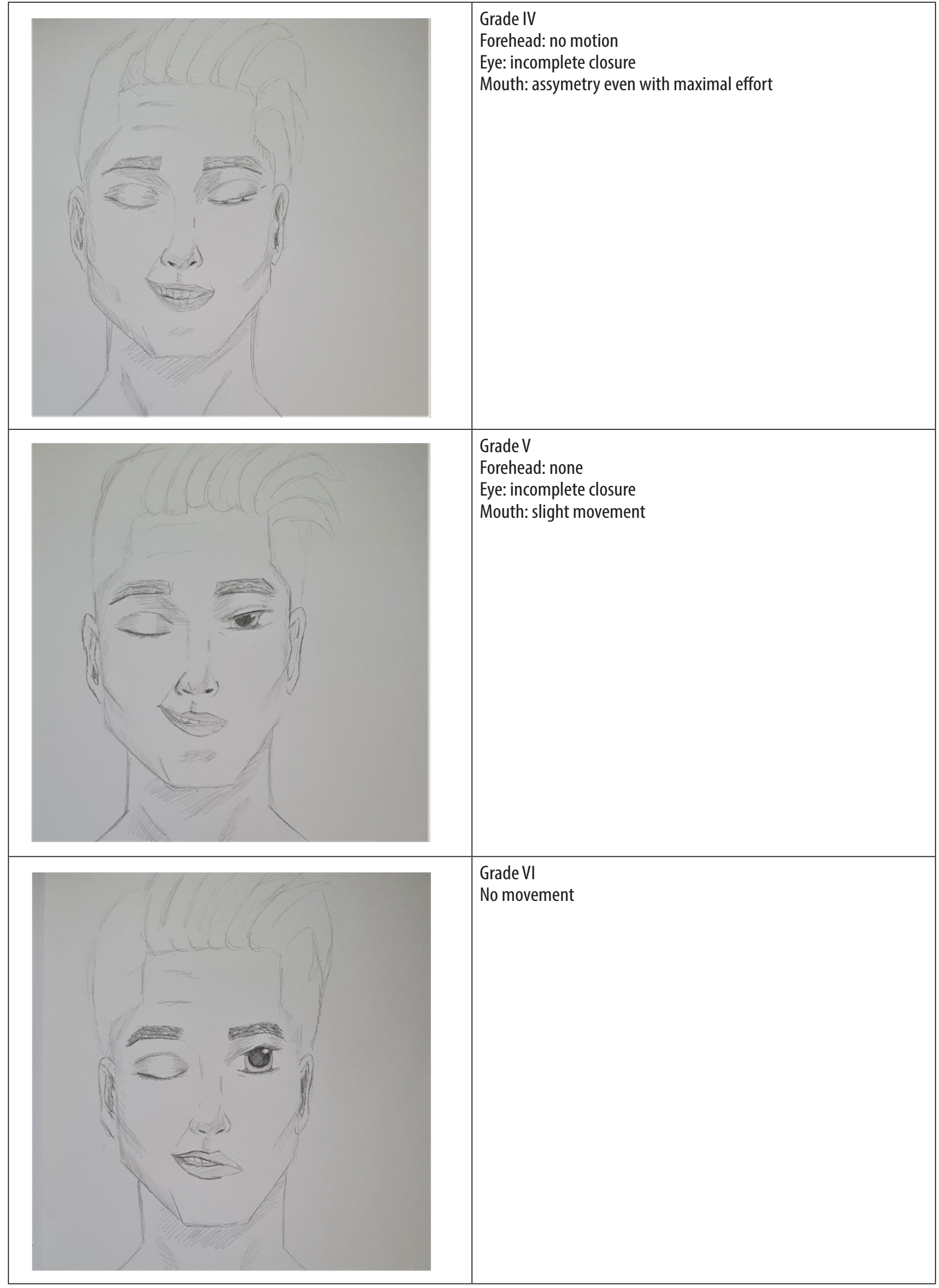


In the first reported case, the aetiology of the palsy was not obvious, clinical manifestation of VZV reactivation at first was not prominent, suggested only by ear pain, with no abnormalities in otoscopy. In the second case, vesicular lesions were prominent, restricted to two dermatomes and left no clinical uncertainty.

VZV reactivation in children is not as common as in adults and the symptoms are usually mild. Children who has acquired infection prenatally or in the first 12 months of life are at increased risk of reactivation. Pain or pruritus in the affected dermatome, fever and malaise can appear prior to or simultaneously with appearance of vesicles. New lesions usually stop building up within 3 to 7 days (earlier if antiviral treatment is administered) and are followed by crusting [1]. Skin eruptions are generally unilateral and may be accompanied by regional lymphadenopathy. Vesicles can form also on mucous membranes, so meticulous examination of the eyes and mouth is indicated. Zoster sine herpete is VZV reactivation without skin lesions and has been associated with idiopathic facial palsy [1]. Children rarely experience post-herpetic neuralgia (pain persisting longer than 1 month) [5]. Typically, diagnosis is based on clinical presentation and requires no additional testing. Antibody testing ( $\operatorname{IgG}$ and $\operatorname{IgM}$ ) can be used to determine the serological status if patient's history of varicella/varicella immunisation is unknown [1]. Polymerase chain reaction (PCR) of vesicular fluid is useful to distinguish between vaccine and wild-type strains [5].

Most commonly used clinical tool for assessment of the facial nerve function is House Brackmann scale. This grading system can be used irrespectively of the etiology. The visual description of this classification is presented in the Table 1.

In the study of Boemo et al., among adult patients with Ramsay-Hunt syndrome, grade IV + grade V paralysis constituted $77 \%$ of all cases. Moreover, at 6-month follow-up examination, all patients with level II-III palsy recovered to level I, whereas only $14 \%$ of level V patients recovered to level I [4]. Yeo et al. assessed that recovery rates in Bell's palsy were better than in Ramsay-Hunt Syndrome (for grades II or better in House Brackmann scale it was $96.3 \%$ to $84.6 \%$ ). According to this study, in the Ramsay-Hunt syndrome, young age and lower initial level of palsy were good prognostic factors [6]. Presumably House-Brackmann scale is not only a descriptive tool, but one of a prognostic value.

Observed tendencies correlate with our series of cases. Our second case, who initially had lower level of facial palsy, has recovered more quickly.

Basic treatment consists of acyclovir and glucocorticosteroids. In the case of neurological symptoms in the course of herpes zoster, acyclovir should be administered intravenously in the dose of $10 \mathrm{mg} / \mathrm{kg}$ body weight $3 \times /$ day for $7-10$ days $[7,8]$.
Anti-inflammatory properties of steroids are well known and used in day-to-day medical practice. As inflammation and oedema of the nerve are the most probable cause of facial palsy, corticosteroids seem to be the drugs of choice in order to prevent irreversible nerve damage. Madhok et al. evaluated 7 studies (895 participants) that brought evidence of moderate to high quality in favour of treating facial palsy with corticosteroids [9].

According to De Rue and van Benthem, combination of antiviral drugs and corticosteroids improves the results of treatment [10]. In the study of da Costa Monsanto, $51.4 \%$ of patients graded initially as House Brackmann $\mathrm{V}$ to VI who received combined therapy (antivirals and steroids) has improved to grade I or II [11].

Various methods are applied additionally to the standard therapy in order to stimulate the recovery of the nerve and improvement of its function. Most popular are physical therapy, laser therapy, phototherapy, massages, thermotherapy, acupuncture and electrotherapy. According to the review of 12 studies, there is only low quality evidence that facial exercises can be of benefit and no evidence behind other types of therapy exists [12].

Ramsay-Hunt syndrome is a rare disease in children and the number of studies assessing progression of the disease, treatment and prognosis is limited. Results of the studies about treatment of RHS are ambiguous, but taking into consideration the risk of lifelong facial paralysis and hearing loss as an alternative, combination of antiviral and corticosteroid therapy should be recommended. No universal paediatric guidelines and regimens are available at the time of writing this article. Further research is warranted in order to standardise the treatment.

\section{DISCLOSURE}

The authors declare no conflict of interest.

\section{REFERENCES}

1. Arvin AM. Varicella Zoster Virus. Facial Nerve Palsy. In: Long Sarah S. Principles and practice of pediatric infectious diseases. Elsevier Saunders-Philadelphia 2012; 1035-1041.

2. Bienkowski C, Kowalczyk M, Talarek E, et al. Meningitis and Ramsay-Hunt syndrome in a 17 -year old girl. Neuroendocrinol Lett 2019; 40: 149-151.

3. Serhan D, Hatice D, Murat S, et al. A Pediatric Case of Ramsay Hunt Syndrome. Case Rep Otolaryngol 2014; 469565.

4. Boemo RL, Navarrete ML, Garcia-Arumi AM, et al. Ramsay Hunt syndrome: our experience. Acta Otorrinolaringol Esp 2010; 61: 418-421.

5. Kronman MP. Infectious diseases - Varicella Zoster Virus Infection. In: Marcdante KJ, Kliegman RM Nelson Essentials of Pediatrics. Elsevier-Philadelphia 2019; 378-379.

6. Yeo SW, Lee DH, Jun BC, et al. Analysis of prognostic factors in Bell's palsy and Ramsay Hunt Syndrome. Auris Nasus Larynx 2007; 34: 159-164.

7. Talarek E, Marczyńska M, Lipińska M. Półpasiec u dzieci. Od objawów do leczenia. Forum Pediatrii Praktycznej 2018; 20: 26-29. 
8. Szenborn L, Kraszewska-Głomba B, Jackowska T, et al. Polish consensus guidelines on the use of acyclovir in the treatment and prevention of VZV and HSV infections. J Infect Chemother 2016; 22: 65-71.

9. Madhok VB, Gagyor I, Daly F, et al. Corticosteroids for Bell's palsy (idiopathic facial paralysis). Cochrane Database of Systematic Reviews 2016; 7: CD001942.

10. de Ru JA, van Benthem PP. Combination therapy is preferable for patients with Ramsay Hunt syndrome. Otol Neurotol 2011; 32: 852-855.

11. Monsanto R, Bittencourt AG, Neto NJ, et al. Treatment and prognosis of facial palsy on Ramsay Hunt syndrome: results based on a review of the literature. Int Arch Otorhinolaryngol 2016; 20: 394-400.

12. Teixeira LJ, Valbuza JS, Prado GF. Physical therapy for Bell's palsy (idiopathic facial paralysis). Cochrane Database of Systematic Reviews 2011; 12: CD006283. 